\title{
Determination of cardiovascular dysfunction before initiation of extracorporeal membrane oxygenation in septic shock
}

\author{
Nathalie Van Der Rijst ${ }^{1} \cdot$ Chirantan Mangukia ${ }^{2}$ (D) Nadeem Muhammad ${ }^{2} \cdot$ Gengo Sunagawa $^{2}$. \\ Stacey Brann ${ }^{2}$. Yoshiya Toyoda ${ }^{2}$
}

Received: 29 October 2020 / Revised: 4 December 2020 / Accepted: 8 December 2020 / Published online: 26 January 2021

(C) Indian Association of Cardiovascular-Thoracic Surgeons 2021

\begin{abstract}
Extracorporeal membrane oxygenation (ECMO) is being increasingly used in patients having sepsis-induced cardiovascular dysfunction. We report successful use of venovenous ECMO in septic shock secondary to pneumonia in the presence of severe left ventricular dysfunction. We also discuss the quantitative evaluation of cardiovascular dysfunction, which provides important input in choosing the type of ECMO in septic shock.
\end{abstract}

Keywords Extracorporeal membrane oxygenation $\cdot$ Venovenous $\cdot$ Septic shock $\cdot$ Cardiovascular dysfunction

\section{Introduction}

Usage of extracorporeal membrane oxygenation (ECMO) in septic shock is increasingly reported. Mostly, septic shock is vasodilatory, but recently a concept of sepsis-induced cardiovascular dysfunction is gaining popularity. In the presence of cardiovascular dysfunction, venoarterial ECMO is almost always preferred. However, determination of pre-ECMO cardiovascular dysfunction is not very clear and varies with institutional practice. We report a successful case of venovenous ECMO in a patient with severe septic shock secondary to pneumonia in the presence of pre-existing severe cardiac dysfunction.

\section{Case report}

A 56-year-old-male presented to an outside facility with complaints of dyspnea and acutely worsening chest discomfort. His past medical history was significant for recent coronavirus

Chirantan Mangukia

chirantan.mangukia@outlook.com

1 Department of Thoracic Medicine and Surgery, Temple University Hospital, Philadelphia, PA, USA

2 Division of Cardiovascular Surgery, Temple University Hospital, 3401 N. Broad Street, 3rd Floor, Parkinson Pavilion,

Philadelphia, PA 19140, USA disease (COVID) pneumonia (3 months ago) superimposed on moderate centrilobular emphysema and controlled human immunodeficiency virus (HIV) infection on antiretroviral therapy, as well as ischemic cardiomyopathy with left ventricular (LV) ejection fraction of 15-20\%, requiring a bi-ventricular implantable cardioverter-defibrillator (ICD) and atrial fibrillation on chronic anticoagulation. In the emergency room, an unenhanced computed tomography (CT) scan of chest demonstrated consolidative ground-glass opacities distributed in the right upper and lower lobes, superimposed on underlying emphysema. His laboratory work was significant for leukocytosis with left shift, mild lactic acidosis, acute kidney injury (AKI), and an international normalized ratio (INR) of 3.7. The patient became progressively hypotensive and was therefore transferred to our center's intensive care unit (ICU) after initiation of vasopressors, broad-spectrum antibiotics, and high-dose corticosteroids.

Within $24 \mathrm{~h}$ of his ICU admission, the patient became increasingly somnolent and was subsequently intubated for airway protection. Testing revealed a positive Legionella antigen in urine. Also, his X-ray chest significantly worsened from his admission with almost complete white-out of the right lung (Fig. 1). Despite optimum ventilation and prone positioning, the patient developed refractory hypoxemia with hypercarbia with combined respiratory and metabolic acidosis. Arterial blood gas $(\mathrm{ABG})$ analysis revealed $\mathrm{pH}$ of 7.23 , $\mathrm{PCO}_{2}$ of $57, \mathrm{PO}_{2}$ of 62 , base deficit of -5 , and oxygen saturation $86 \%$ on ventilator settings of assisted volumecontrolled mode with tidal volume of $450 \mathrm{cc}$, respiratory rate 


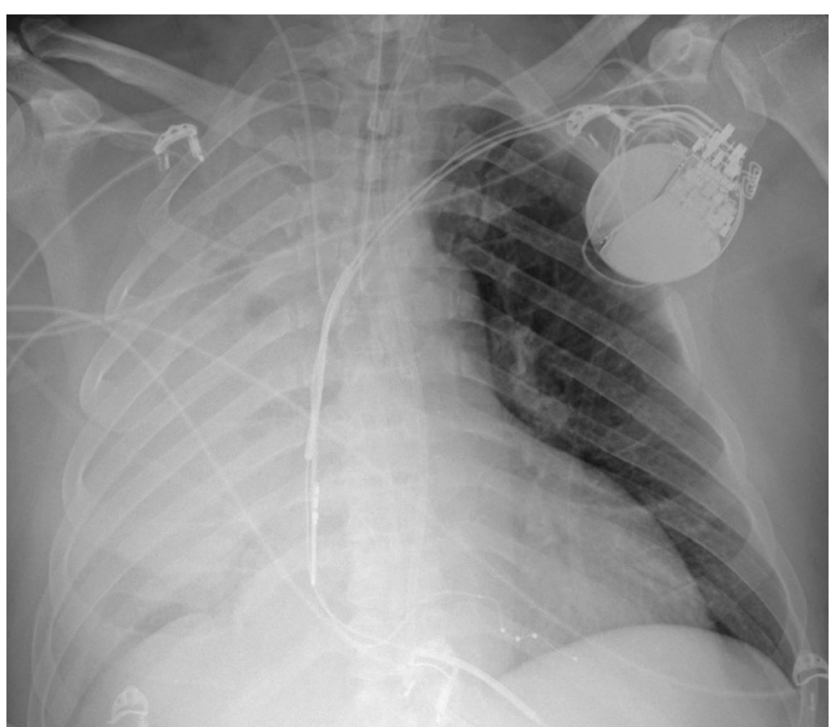

Fig. 1 Chest X-ray suggesting right-sided white-out secondary to pneumonia and recurrent mucus plugging

of $30, \mathrm{FiO}_{2}$ of $100 \%$, and positive end-expiratory pressure (PEEP) of $15 \mathrm{~mm}$ of $\mathrm{Hg}$. His mean arterial pressure was $59 \mathrm{mmHg}$ with central venous pressure (CVP) of 16$18 \mathrm{mmHg}$ on maximum doses of four vasopressors (epinephrine at $10 \mathrm{mcg} / \mathrm{min}$, norepinephrine at $30 \mathrm{mcg} / \mathrm{min}$, phenylephrine at $300 \mathrm{mcg} / \mathrm{min}$, and vasopressin at 0.04 units $/ \mathrm{min}$ ). His AKI also worsened, requiring initiation of continuous venovenous hemodialysis (CVVH), along with worsening lactatemia of $3.8 \mathrm{mmol} / \mathrm{L}$.

Clearly, existing respiratory support level was not adequate for him. Since he was only 56 years old, very compliant to antiretroviral medicines, and had a potentially reversible cause, it was decided to give him a chance by escalating the support level to ECMO.

The dilemma was whether his heart function was adequate or not-i.e., a choice between venoarterial (VA) or venovenous (VV) ECMO. Bedside echocardiography (Video 1) suggested ejection fraction of 15 to $20 \%$ with severe diffuse hypokinesis with grade 2 diastolic dysfunction. Left ventricular end-diastolic dimension was $70 \mathrm{~mm}$ and end systolic dimension was $61 \mathrm{~mm}$. There was trivial mitral regurgitation with restricted posterior leaflet mobility. The tricuspid valve was found to be normal and no regurgitation was identified. The right ventricle was mild to moderately dilated and systolic function was mildly reduced by visual assessment. Tricuspid annular plane systolic excursion (TAPSE) was $2.3 \mathrm{~cm}$. Lactatemia, new onset of acute kidney injury requiring CVVH (end-organ dysfunction), poor LV function, and high inotropes/pressor requirement were pointing toward marginal cardiac output. Because of his high INR, putting a large bore venous sheath to advance the Swan-Ganz catheter was deemed to be high risk, as well as unnecessary, as thermodilution in the presence of continuous fever would have been unreliable. So, we decided to utilize right-sided central venous line/triple-lumen catheter to draw blood representing mixed venous blood gas. It showed saturation of $59 \%$, against systemic saturation of $86 \%$ in arterial blood gas. Utilizing Fick's formula, we came up with cardiac index as $2.9 \mathrm{~L} / \mathrm{min} / \mathrm{m}^{2}$ and it was considered adequate in multidisciplinary discussion.

Therefore, the decision was made to continue with VV ECMO using percutaneous groin-groin configuration. A 60$\mathrm{cm}$-long 25 French multistage drainage cannula (Medtronic Biomedicus - 96880-025) was inserted into the left common femoral vein and was fixed at $35 \mathrm{~cm}$ at skin level. A 50-cmlong 17 French return cannula (Medtronic Biomedicus 96670-017, with multiple holes at near tip only) was inserted into the right common femoral vein and was fixed at $50 \mathrm{~cm}$ at skin level (Fig. 2). ECMO flow was initiated at $4 \mathrm{~L} / \mathrm{min}$ and 3700 RPM on Rotaflow Centrifugal Pump System with sweep gas of $3 \mathrm{~L}$ and sweep $\mathrm{FiO}_{2}$ of $100 \%$. A contingency plan was also devised to switch to VA ECMO if the patient did not improve. Immediately after initiation of VV ECMO, his oxygen saturation increased to $100 \%$ and his $\mathrm{ABG}$ improved to a $\mathrm{pH}$ of 7.42, $\mathrm{PCO}_{2}$ of 32, and $\mathrm{PO}_{2}$ of 304 with base deficit of 3. Lung protective mechanical ventilation was initiated with lower PEEP and $\mathrm{FiO}_{2}$ of $30 \%$. Pre-ECMO biochemistry suggested C-reactive protein (CRP) of $38.3 \mathrm{mg} / \mathrm{dL}$, D-dimer of $3496 \mathrm{ng} / \mathrm{mL}$, fibrinogen of $647 \mathrm{mg} / \mathrm{dL}$, INR of 3.8 , activated partial thromboplastin time (aPTT) of $105.8 \mathrm{~s}$, serum ferritin of $411 \mathrm{ng} / \mathrm{ml}$, creatinine kinase of $536 \mathrm{U} / \mathrm{L}$, and lactate dehydrogenase (LDH) of $943 \mathrm{U} / \mathrm{L}$. We continued to follow inflammatory markers and hemolysis labs until he was decannulated. His highest D-dimer level was $6156 \mathrm{ng} / \mathrm{mL}$ on day 5 , following that, it started improving. Highest LDH was $1759 \mathrm{U} / \mathrm{L}$ on

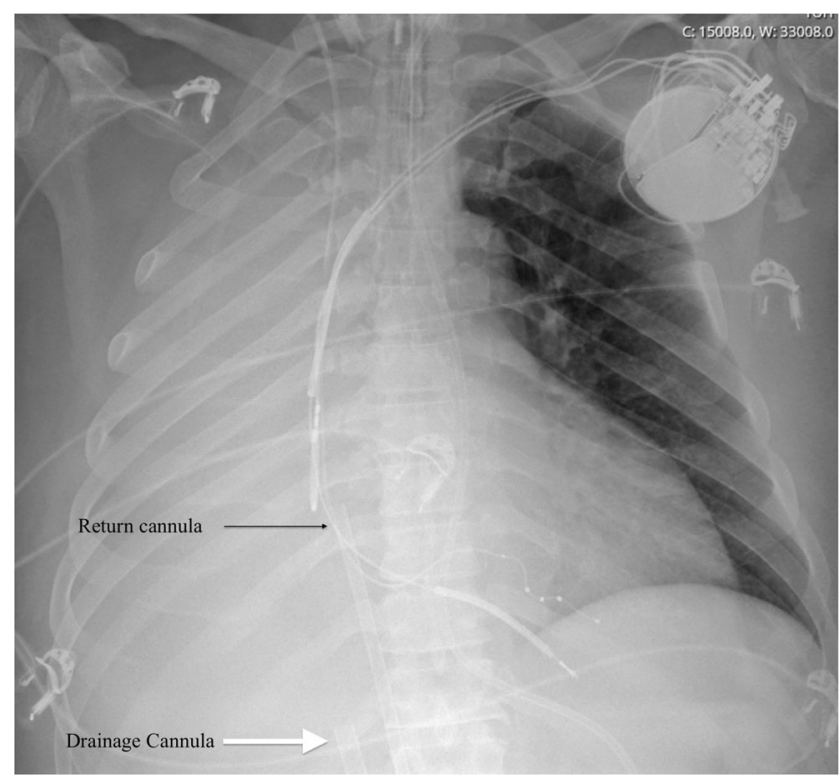

Fig. 2 Chest X-ray showing VV ECMO cannula positions: black arrow suggests return cannula, white thick arrow suggests drainage cannula 
day 11 which was thought to be appropriate elevation given his high baseline LDH. One unit of blood was given to keep hemoglobin above $7 \mathrm{~g} / \mathrm{dL}$. A total of 6 units of fresh frozen plasma was infused intermittently during 18 days of ECMO support in order to correct the INR as needed. A total of 6 units of platelets was transfused to correct thrombocytopenia (secondary to anti-retroviral medicines and sepsis) less than $50,000 \mathrm{per} / \mathrm{mm}^{3}$. Over the next several days, his pressor requirements continued to trend down slowly, and his lactic acidosis resolved.

Although the patient's condition improved, he did have a prolonged hospital course. He required daily bronchoscopies due to recurrent mucus plugging. A percutaneous tracheostomy was placed 13 days after intubation; and 18 days after initiation of VV ECMO, the patient was successfully decannulated. His hospital course was further complicated by Clostridium difficile colitis, methicillin-resistant Staphylococcus aureus bacteremia, encephalopathy secondary to septic emboli and a subacute infarct in middle cerebral artery territory, and critical illness polyneuropathy. Approximately a month after ECMO decannulation, CT chest suggested unchanged centrilobular emphysema and small bilateral pleural effusions. Ultimately, the patient was discharged to a long-term acute care facility on day 52 of hospitalization.

\section{Discussion}

In USA alone, sepsis is responsible for 1.7 million admissions to hospitals annually with estimated mortality of 35 to $50 \%$ in the presence of septic shock [1]. Transiently reversible myocardial dysfunction is now a very well-known and common feature during septic shock, affecting 25 to $65 \%$ of patients [2-4]. Mortality of the patients with severe myocardial dysfunction and decreased cardiac index exceeds $80 \%$ according to several reports $[2,3]$.

Because of encouraging results in children with survival rates approaching up to $75 \%, \mathrm{ECMO}$ is included as one of the therapeutic options for septic shock in the latest pediatric guidelines [5]. Despite remaining controversial, use of VA ECMO in adults with septic shock refractory to conventional treatment has increased significantly in the last few years, with growing experience from high-volume ECMO centers.

However, there is no universal guideline or consensus published in the literature to determine cardiovascular dysfunction in cases of septic shock. Usually, determination of severity of cardiovascular dysfunction is done using the following four parameters - low cardiac index, left ventricular dysfunction, lactatemia, and high inotropic/vasopressor requirement $[6,7]$. Criteria vary across the institutes. Septic shock is predominantly distributive and therefore definition of cardiovascular dysfunction in such situation is debatable. Some [6] consider a cardiac index less than $3 \mathrm{~L} / \mathrm{min} / \mathrm{m}^{2}$ (considering high output failure physiology), while others [7] consider cardiac index less than $2 \mathrm{~L} / \mathrm{min} / \mathrm{m}^{2}$ as a cutoff level for cardiovascular dysfunction in septic shock. There are also different inotrope/ vasopressor scores available to determine the severity of shock [6,7], but those scores are based on institute-specific inotrope/pressor usage and may not include all the inotropes/ vasopressors available in the market.

Oftentimes, in this situation, objective evidence of cardiac output by using pulmonary artery or transpulmonary thermodilution, noninvasive arterial contour analysis, echocardiography, etc., is not reliable. One can also argue that in the presence of vasodilatory shock and extremely variable tissue oxygen extraction, given the pyrexia is taken into the picture, mixed venous saturation (Fick's method) may also be considered relatively unreliable.

Therefore, in the presence of pneumonia causing septic shock along with cardiorespiratory dysfunction, choice of ECMO becomes trickier. $\mathrm{CO}_{2}$ retention along with lactatemia results in acidemia, which negatively impacts vascular tone, cardiac contractility, and efficiency of inotropes/vasopressors. Oftentimes, patients are ventilated with very high PEEP, which also negatively impacts the cardiac output. In this situation, VV ECMO should help favorably in terms of myocardial contractility, cardiac output, vascular tone, and vasopressor requirements, as it corrects the gas exchange and acidemia and allows for lung-protective ventilation with minimal PEEP.

The vital question remains: can we get away with fixing the gas exchange only with VV ECMO support? In other words, is the improvement achieved by correcting acidemia and reducing PEEP sufficient to improve cardiac output to a level good enough for recovery? VA ECMO is certainly a higher level of circulatory support, and also involves more complications arising from anticoagulation, thromboembolism, and limb ischemia. It also requires a trip to the operating room at the time of decannulation. According to a recent study, when needed, VA ECMO is used much more frequently than VV ECMO for septic shock resulting from the lungs as a primary source [7].

Usage of VV ECMO in severe acute respiratory distress syndrome (ARDS) is very well known [8]. There are several publications providing contemporary guidelines and fundamentals for clinicians for bedside troubleshooting $[8,9]$. Our case was special as it involved severe preexisting cardiac dysfunction also. We decided to help the patient with VV ECMO after calculating cardiac index using Fick's formula and also had a backup plan to switch to VA ECMO in case VV ECMO was found to be inadequate on careful monitoring. At our hospital, we have been consistently using Fick's method which has given us reliable information in determining the type of ECMO. We believe that mixed venous saturation reflects the true physiological requirement of cardiac output, with fair accuracy in such conditions. 
Ours is an aggressive and high-volume center for ECMO. Our patient had a combination of dilated cardiomyopathy with poor LV function, along with septic/distributive shock secondary to respiratory foci. Based on our experience, the decision for VV ECMO turned out to be adequate in reversing cardiovascular dysfunction in septic shock.

A PubMed search, with MeSH words "ECMO" and "Septic shock" retrieved 115 papers. They were all relevant studies, describing VA ECMO support: most describing usage in pediatric population and only one of them described usage of VV ECMO in a small number of adults. Our case revises and reiterates important physiology lessons in a relatively new era of ECMO usage in sepsis.

\section{Conclusion}

Determination of cardiovascular dysfunction in the presence of septic shock is a very important step in choosing the type of ECMO support. No specific guideline or consensus exists for such measurement. Calculating cardiac output using Fick's formula appears to be a helpful marker for such situations in our opinion.

Supplementary Information The online version contains supplementary material available at https://doi.org/10.1007/s12055-020-01119-4.

Funding The authors do not have any pertinent funding disclosure to describe.

\section{Compliance with ethical standards}

Conflict of interest The authors declare that they have no conflict of interest.

Research involving human participants and/or animals Not applicable as per institutional ethical committee as the scientific information presented in paper does not fall into category of clinicals trial or usage of experimental modalities.

Informed consent Obtained.

\section{References}

1. Rhee C, Dantes R, Epstein L, et al. Incidence and trends of sepsis in US hospitals using clinical vs claims data, 2009-2014. JAMA. 2017;318:1241-9.

2. Jeong HS, Lee TH, Bang CH, Kim JH, Hong SJ. Risk factors and outcomes of sepsis-induced myocardial dysfunction and stressinduced cardiomyopathy in sepsis or septic shock: A comparative retrospective study. Medicine. 2018;97:e263.

3. Parker MM, Suffredini AF, Natanson C, Ognibene FP, Shelhamer $\mathrm{JH}$, Parrillo JE. Responses of left ventricular function in survivors and nonsurvivors of septic shock. J Crit Care. 1989;4:19-25.

4. Cioccari L, Luethi N, Weber U, Hilton A, Takala J, Bellomo R. The native cardiac output in human sepsis: a systematic review. Crit Care Resusc. 2016;18:148-56.

5. Davis AL, Carcillo JA, Aneja RK, et al. American College of Critical Care Medicine clinical practice parameters for hemodynamic support of pediatric and neonatal septic shock. Crit Care Med. 2017;45: 1061-93.

6. Bréchot N, Hajage D, Kimmoun A, et al. Venoarterial extracorporeal membrane oxygenation to rescue sepsis-induced cardiogenic shock: a retrospective, multicentre, international cohort study. Lancet. 2020;396:545-52.

7. Falk L, Hultman J, Broman LM. Extracorporeal membrane oxygenation for septic shock. Crit Care Med. 2019;47:1097-105.

8. Patel B, Chatterjee S, Davignon S, Herlihy JP. Extracorporeal membrane oxygenation as rescue therapy for severe hypoxemic respiratory failure. J Thorac Dis. 2019;11:S1688-97.

9. Squiers JJ, Lima B, DiMaio JM. Contemporary extracorporeal membrane oxygenation therapy in adults: Fundamental principles and systematic review of the evidence. J Thorac Cardiovasc Surg. 2016;152:20-32.

Publisher's note Springer Nature remains neutral with regard to jurisdictional claims in published maps and institutional affiliations. 